\title{
The hyperdense MCA sign and the MCA dot sign
}

\section{N Mahomed, $M B$ BCh, FCRad (D)}

Department of Radiology, University of the Witwatersrand, Johannesburg

Corresponding author: N Mahomed (nasreen.mahomed@wits.ac.za)

The hyperdense middle cerebral artery sign (HMCA), first described in 1983, refers to the hyperattenuation of the middle cerebral artery (MCA) M1 segment on non-enhanced computed tomography (CT). ${ }^{1,2}$ The sign is due to a thromboembolus of the M1 segment of the MCA. Because the sign is a marker of vascular occlusion rather than a direct image of the resulting parenchymal changes, the HMCA sign can be considered an indirect indicator of subsequent infarction and is one of the earliest signs of ischaemic stroke. ${ }^{3}$

The MCA dot sign is a punctate focus of hyperattenuation located in the sylvian fissure on non-enhanced CT, and is a recently described variant of the HMCA sign. ${ }^{3}$ The sign represents a thromboembolus within a segmental branch of the MCA, M2 or M3 segment, located within the sylvian fissure. ${ }^{3}$ As the M2 and M3 segmental vessels tend not to course in the transverse plane of imaging, the occluded vessel is seen in cross section, appearing as a hyperattenuating dot within the sylvian fissure. ${ }^{3}$

While the HMCA sign and MCA dot sign are similar in that they both depict thromboembolus at different levels of the MCA, there are important clinical and prognostic distinctions. The HMCA sign suggests that a major cerebral vessel is occluded, suggesting a larger territory at risk for hypoperfusion compared with the more distal vessel occlusion of the MCA dot sign. Therefore, the MCA dot sign in the absence of the HMCA sign is associated with improved short-term clinical outcome. $^{3}$

These signs have a high specificity of almost $100 \%$ with a high positive predictive value but a low sensitivity of approximately $38-40 \%$ for thromboembolic occlusion of the MCA. ${ }^{1-3}$

Mimics of the HMCA sign, the pseudo hyperdense MCA sign, include vascular calcification, raised haematocrit, intravenous contrast and partial volume averaging. ${ }^{1}$ Another important cause of a pseudo

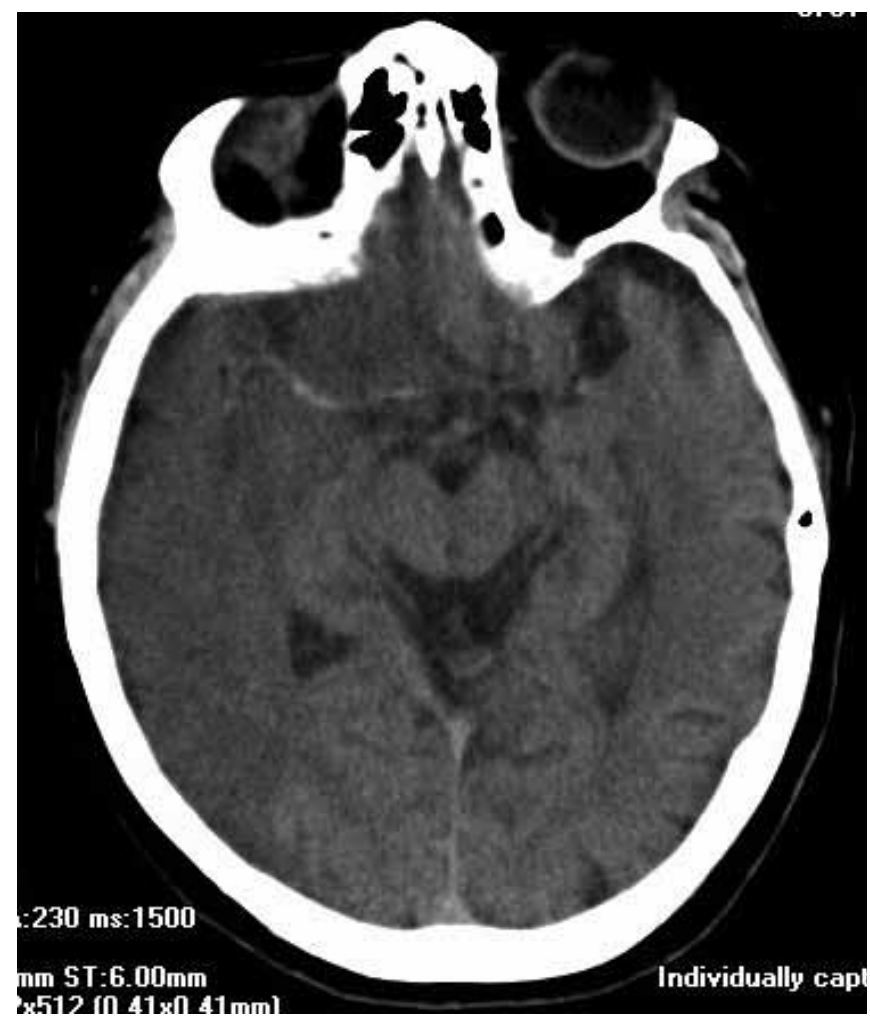

Fig. 1. HMCA sign. Non-enhanced axial CT brain (at the level of the suprasellar cistern) in a patient with sudden onset left hemiparesis demonstrates hyperattenuation (measuring $82 \mathrm{HU}$ ) of the right middle cerebral artery, which is suggestive of occlusion of this artery and is an indirect sign. There is also diffuse hypodensity of the right temporal lobe, a direct sign of ischaemic stroke. 


\section{SIGNS}

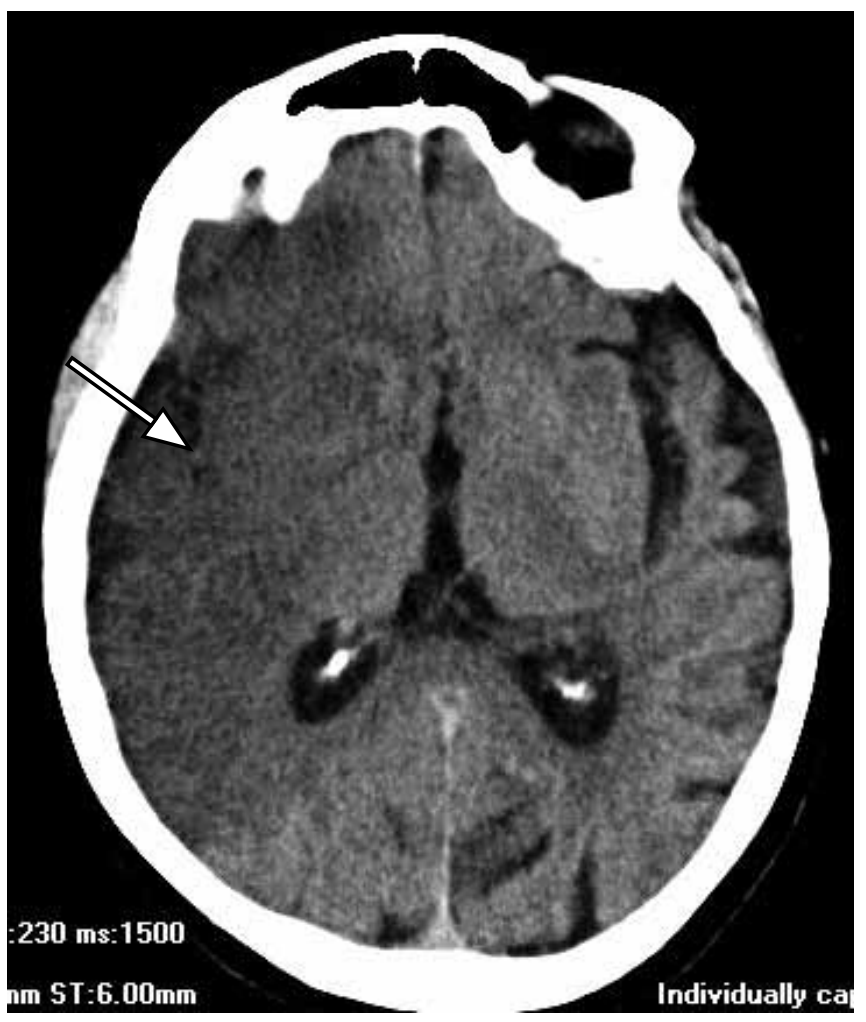

Fig. 2. Non-enhanced axial CT brain of the same patient (at the level of the sylvian fissures) demonstrates a hyperattenuating dot (arrow) in the partially attenuated right sylvian fissure, which is more opaque (measuring $70 \mathrm{HU}$ ) than any structure in the ipsilateral or contralateral sylvian fissure - the MCA dot sign.

hyperdense MCA sign is a normal density MCA appearing hyperdense owing to adjacent abnormal brain parenchymal hypodensity. This may appear in the setting of infection, tumour or contusion. ${ }^{1}$ In a study by Koo et al. to establish the objective criteria for the HMCA sign, the authors concluded that an absolute attenuation value of 43 Hounsfield units (HU) of the abnormal MCA and a ratio of more than 1.2 between the abnormal MCA attenuation compared with the normal contralateral MCA attenuation, identified all the hyperdense MCAs associated with acute ischaemic stroke. ${ }^{4}$

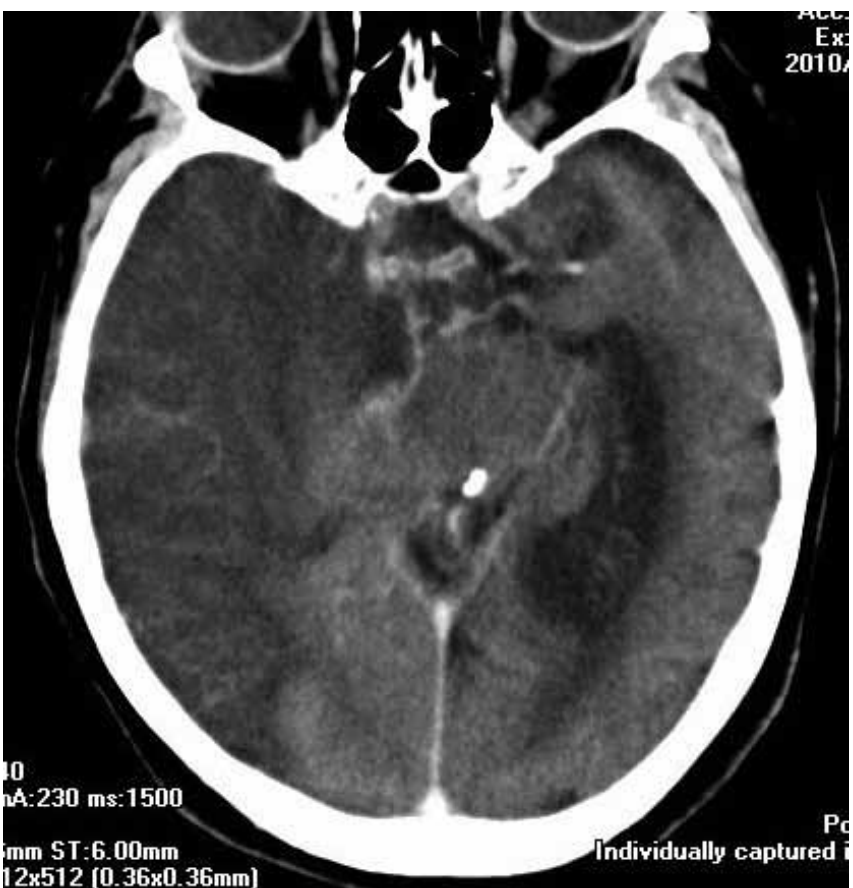

Fig. 3. Enhanced axial CT brain done 5 days later (at the level of the suprasellar cistern) demonstrates evolution of the complete right MCA ischaemic stroke with significant mass effect and features of luxury perfusion.

Non-enhanced CT of the brain is usually the first diagnostic study performed for the investigation of acute neurologic symptoms and signs as it is sensitive for haemorrhage and less expensive than MR. Early non-enhanced CT scans can be an invaluable tool in detecting these indirect signs of acute infarction and are helpful for accurate and prompt management of these patients. ${ }^{3}$

1. Jha B, Kothari M. Pearls \& Oysters: Hyperdense or pseudohyperdense MCA sign: A Damocles sword? Neurology 2009;72:el16-e117.

2. Chavhan GB, Shroff MM. Twenty classic signs in neuroradilogy: A pictorial essay. Indian J Radiol Imaging 2009;19:135-145.

3. Shetty SK. The MCA dot sign. Radiology 2006;241:315-318.

4. Koo CK, Teasdale E, Muir KW. What constitutes a true hyperdense middle cerebral artery sign Cerebrovasc Disease 2000;10:419-423. 